\title{
On stability analysis and existence of positive solutions for a general non-linear fractional differential equations
}

\author{
Amita Devi ${ }^{1}$, Anoop Kumar ${ }^{1}$, Dumitru Baleanu ${ }^{2,3,4^{*}}$ (D) and Aziz Khan ${ }^{5}$
}

\section{"Correspondence:}

dumitru@cankaya.edu.tr

${ }^{2}$ Department of Mathematics,

Faculty of Art and Sciences, Cankaya

University, Cankaya, Turkey

${ }^{3}$ Institute of Space Science,

Magurele-Bucharest,

Magurele-Bucharest, Romania

Full list of author information is

available at the end of the article

\begin{abstract}
In this article, we deals with the existence and uniqueness of positive solutions of general non-linear fractional differential equations (FDEs) having fractional derivative of different orders involving p-Laplacian operator. Also we investigate the Hyers-Ulam (HU) stability of solutions. For the existence result, we establish the integral form of the FDE by using the Green function and then the existence of a solution is obtained by applying Guo-Krasnoselskii's fixed point theorem. For our purpose, we also check the properties of the Green function. The uniqueness of the result is established by applying the Banach contraction mapping principle. An example is offered to ensure the validity of our results.
\end{abstract}

MSC: 26A33; 34BB2;45ND5

Keywords: Hyers-Ulam stability; p-Laplacian operator; Caputo fractional derivative; Guo-Krasnoselskii's fixed point theorem; EU of positive solutions

\section{Introduction}

Fractional calculus concerns the applications of derivatives and integrals of arbitrary order. During the last few decades, it received great attention because of its various applications in diverse scientific fields. Arbitrary-order models are more flexible than integerorder models. FDEs arise in numerous scientific and engineering fields such as physics, polymer rheology, geophysics, biophysics, aerodynamics, capacitor theory, biology, nonlinear oscillation of earthquake, control theory, blood flow phenomena, viscoelasticity, and electrical circuits. For the exhaustive study of its applications, we refer to extensive work [1-7]. The fundamental differences between exponential decay, the power law, the Mittag-Leffler law and some possible applications in nature are presented in [8, 9].

Nowadays, the existence and uniqueness (EU) of solutions for different type of FDEs is a field of intensive research. Here, we introduce some important and recent work of several researcher about the existence of a positive solution (EPS) of different classes of FDEs. For example, the EU results for Dirichlet and mixed problems of singular FDEs with the Riemann-Liouville sense of fractional derivative were investigated by Agarwal et al. $[10,11]$. Baleanu et al. in [12] established the existence of a solution on partially ordered

(c) The Author(s) 2020. This article is licensed under a Creative Commons Attribution 4.0 International License, which permits use, sharing, adaptation, distribution and reproduction in any medium or format, as long as you give appropriate credit to the original author(s) and the source, provide a link to the Creative Commons licence, and indicate if changes were made. The images or other third party material in this article are included in the article's Creative Commons licence, unless indicated otherwise in a credit line to the material. If material is not included in the article's Creative Commons licence and your intended use is not permitted by statutory regulation or exceeds the permitted use, you will need to obtain permission directly from the copyright holder. To view a copy of this licence, visit http://creativecommons.org/licenses/by/4.0/. 
Banach spaces for a non-linear FDEs. Vong studied the singular FDEs involving non-local type boundary conditions in [13] by using fixed point techniques. For more details of EU of different types of FDEs with different types of fractional derivatives, see [14-35]. Numerical solutions for the fractional Fisher's type equations involving the Atangana-Baleanu fractional derivative by methods of spectral collocation are in [26]. As of lately, some authors investigated the FDEs with p-Laplacian operator by diverse types of mathematical techniques. For instance, Khan et al. investigated the existence criterion for solutions for FDEs involving the $\phi_{p}$-Laplacian operator in [36]. The EU of results for FDEs with $\phi_{p^{-}}$ Laplacian operator are analyzed by Chuanzhi Bai in [37] via fixed point theorems. Also we present the Green function's properties and two examples to illustrate the results. The EPS for FDEs with the $\phi_{p}$-Laplacian operator is studied by Tian et al. [38] and EPSs are obtained with the help of a monotone iterative method. For more EU results for FDEs with a p-Laplacian operator one may refer to [39-42]

Recently, a great interest has been shown in the study of HU stability of non-linear FDEs with different type of boundary conditions. By HU stability we mean that there exists an exact solution very close to the approximate solution of a FDE and that the error can be calculated. The EU of solutions and HU stability FDEs with p-Laplacian operator and ABCfractional derivative involving a spatial singularity is derived by Khan et al. in [43] using the well-known Guo-Krasnoselskii theorem. Khan et al. [44] discussed the analytical study of existence and stability results of a singular non-linear FDEs with $\phi_{p}$-operator involving fractional integral and differential boundary conditions. The EU and HU stability of solutions for a coupled system of FDEs involving the derivative in Caputo's sense are proved by Khan et al. [45] using a Leray-Schauder-type fixed point theorem and topological degree theory. Li et al. investigated the HU stability of FDEs in [46] and also presented an example to illustrate their result. Stability and EU of solutions for the fractional order HIV model were introduced by Khan et al. in [47]. Existence and stability of solutions for singular delay FDEs with fractional integral initial conditions by using the Green function and the fixed point theorem were established by the Khan et al. in [48]. For more details of stability analysis, see [49-57].

Motivated by the above work, we introduce the EU and HU stability results, for nonliner FDEs involving Caputo fractional derivatives of distinct orders with $\phi_{P}^{*}$ Laplacian operator:

$$
\left\{\begin{array}{l}
{ }^{c} \mathscr{D}^{\zeta} \phi_{p}^{*}\left[{ }^{c} \mathscr{D}^{\sigma}\left(\mathfrak{g}(t)-\sum_{i=1}^{m} \lambda_{i}(t)\right)\right]=-\psi^{*}(t, \mathfrak{z}(t)), \quad t \in[0,1], \\
\left.\phi_{p}^{*}\left[\mathscr{D}^{\sigma} \mathfrak{g}(t)-\sum_{i=1}^{m} \lambda_{i}(t)\right]\right|_{t=0}=0, \quad \mathfrak{g}(0)=\sum_{i=1}^{m} \lambda_{i}(0), \\
\mathfrak{g}^{\prime}(1)=\sum_{i=1}^{m} \lambda_{i}^{\prime}(1), \quad \mathfrak{g}^{j}(0)=\sum_{i=1}^{m} \lambda_{i}^{j}(0) \quad \text { for } j=2,3,4, \ldots, n-1,
\end{array}\right.
$$

where ${ }^{c} \mathscr{D}^{\zeta},{ }^{c} \mathscr{D}^{\sigma}$ denotes the derivative of fractional order $\zeta$ and $\sigma$ in Caputo's sense, respectively, and $\psi^{*}, \lambda_{i}(t)$ are continuous functions. The orders $n-1<\sigma \leq n, 0<\zeta \leq 1$ where $n \geq 4, \psi^{*} \in \mathcal{L}[0,1]$ and $\phi_{p}^{*}(\xi)=|\xi|^{p-1} \xi$ denotes the $p$-Laplacian operator and satisfies $\frac{1}{p}+\frac{1}{q}=1,\left(\phi_{p}^{*}\right)^{-1}=\phi_{q}^{*}$. The rest of article is divided in four parts. Basic definitions and desired lemmas are presented in Sect. 1 and properties of the Green functions are discussed in Sect. 2. The EU results are given in Sect. 3. HU stability is discussed in Sect. 4. In Sect. 5, we introduce an example.

Here, we introduce certain definitions, desired lemmas and theorems, which are essential to find the main result. 
Definition 1.1 ([1]) For an integrable and real valued continuous function $\psi^{*}$ defined on $(0,+\infty)$, the Riemann-Liouville integral of fractional order $\delta \in \mathbb{R}$ is defined as

$$
I^{\delta} \psi^{*}(y)=\frac{1}{\Gamma(\delta)} \int_{0}^{y}(y-x)^{\delta-1} \psi^{*}(x) d x, \quad \delta>0
$$

Definition $1.2([1])$ For an n-times continuously differentiable real valued function $\psi^{*}$ defined on $(0,+\infty)$, the Caputo derivative of fractional order $\delta \in \mathbb{R}(\delta>0)$ is defined as

$$
{ }^{c} \mathscr{D}^{\delta} \psi^{*}(y)=\frac{1}{\Gamma(\mathrm{n}-\delta)} \int_{0}^{y}(y-x)^{\mathrm{n}-\delta-1}\left(\psi^{*}\right)^{n}(x) d x, \quad \mathrm{n}-1<\delta<\mathrm{n}, \mathrm{n}=[\delta]+1,
$$

where $[\delta]$ represents the greatest integer and the integral exists on the $(0,+\infty)$ interval.

Lemma 1.1 ([2]) Let $\sigma \in(\kappa-1, \beta]$ and $\psi^{*}(t) \in C^{k-1}$, then

$$
\mathscr{I}^{\sigma} \mathscr{D}^{\sigma} \psi^{*}(y)=\psi^{*}(y)+a_{0}+a_{1} y+a_{2} y^{2}+a_{3} y^{3}+\cdots+a_{k-1} y^{k-1}
$$

for the $a_{j} \in \mathbb{R}$ for $j=0,1,2, \ldots, k-1$.

Theorem 1.2 $\left([58,59]\right.$, Guo-Krasnoselskii theorem) Consider $\Omega^{*}$ to be a Banach space and let a cone $\mathscr{K}^{*} \in \Omega^{*}$. Assume that $\mathcal{A}_{1}^{*}, \mathcal{A}_{2}^{*}$ are two bounded subsets of $\Omega^{*}$ such that $0 \in \mathcal{A}_{1}^{*}, \overline{\mathcal{A}_{1}^{*}} \subset \mathcal{A}_{2}^{*}$. Then an operator $\mathcal{L}^{*}: \mathscr{K}^{*} \cap\left(\overline{\mathcal{A}_{2}^{*}} \backslash \mathcal{A}_{1}^{*}\right) \longrightarrow \mathscr{K}^{*}$, which is completely continuous and satisfies

$$
\left(\mathscr{P}_{1}^{*}\right) \quad\left\|\mathcal{L}^{*} \mathfrak{z}\right\| \leq\|\tilde{z}\| \quad \text { if } \mathfrak{z} \in \mathcal{K}^{*} \cap \partial \mathcal{A}_{1}^{*} \text { and }\left\|\mathcal{L}^{*} \mathfrak{z}\right\| \geq\|\mathfrak{z}\| \quad \text { if } \mathfrak{z} \in \mathcal{K}^{*} \cap \partial \mathcal{A}_{2}^{*} \text {, }
$$

or

$$
\left(\mathscr{P}_{2}^{*}\right) \quad\left\|\mathcal{L}^{*} \mathfrak{z}\right\| \geq\|\mathfrak{z}\| \quad \text { if } \tilde{z} \in \mathcal{K}^{*} \cap \partial \mathcal{A}_{1}^{*} \quad \text { and } \quad\left\|\mathcal{L}^{*} \mathfrak{z}\right\| \leq\|\mathfrak{z}\| \quad \text { if } \tilde{z} \in \mathcal{K}^{*} \cap \partial \mathcal{A}_{2}^{*} \text {, }
$$

has a fixed point in $\mathcal{K}^{*} \cap\left(\overline{\mathcal{A}_{2}^{*}} \backslash \mathcal{A}_{1}^{*}\right)$.

Lemma 1.3 ([44, 45]) For the p-Laplacian operator $\phi_{p}^{*}$, the following conditions hold true:

(1) If $\left|\gamma_{1}\right|,\left|\gamma_{2}\right| \geq \sigma>0,1<p \leq 2, \gamma_{1} \gamma_{2}>0$, then

$$
\left|\phi_{p}^{*}\left(\gamma_{1}\right)-\phi_{p}^{*}\left(\gamma_{2}\right)\right| \leq(p-1) \sigma^{p-2}\left|\gamma_{1}-\gamma_{2}\right|
$$

(2) If $p>2,\left|\gamma_{1}\right|,\left|\gamma_{2}\right| \leq \sigma^{*}>0$, then

$$
\left|\phi_{p}^{*}\left(\gamma_{1}\right)-\phi_{p}^{*}\left(\gamma_{2}\right)\right| \leq(p-1)\left(\sigma^{*}\right)^{p-2}\left|\gamma_{1}-\gamma_{2}\right|
$$

\section{Green function and properties}

Theorem 2.1 Consider $\psi^{*} \in \mathcal{C}[0,1]$ satisfying the FDE with $\phi_{p}^{*}(1.1)$. Then, for $\zeta \in(0,1]$ and $\sigma \in(n-1, n]$, the FDEs (1.1) involving the $\phi_{p}^{*}$ Laplacian operator has a solution equivalent to

$$
\tilde{g}(t)=\sum_{i=1}^{m} \lambda_{i}(t)+\int_{0}^{1} \mathcal{H}^{\sigma}(t, \jmath) \phi_{q}^{*}\left(\frac{1}{\Gamma(\zeta)} \int_{0}^{\lrcorner}(s-\epsilon)^{\zeta-1} \psi^{*}(\epsilon, \mathfrak{g}(\epsilon)) d \epsilon\right) d s,
$$


where the Green function $\mathcal{H}^{\sigma}(t, s)$ is defined by

$$
\mathcal{H}^{\sigma}(t, s)= \begin{cases}\frac{-(t-s)^{\sigma-1}}{\Gamma(\sigma)}+t \frac{(1-s)^{\sigma-2}}{\Gamma(\sigma-1)}, & 0<s \leq t<1 \\ t \frac{(1-s)^{\sigma-2}}{\Gamma(\sigma-1)}, & 0<t \leq s<1 .\end{cases}
$$

Proof Taking the integral operator $I^{\zeta}$ on both sides (1.1) and using Lemma 1.1, Eq. (1.1) becomes

$$
\phi_{p}^{*}\left({ }^{c} \mathscr{D}^{\sigma}\left[\tilde{g}(t)-\sum_{i=1}^{m} \lambda_{i}(t)\right]\right)=-I^{\zeta}\left[\psi^{*}(t, \mathfrak{z}(t))\right]+C_{0} .
$$

From the condition $\left.\phi_{p}^{*}\left({ }^{c} \mathscr{D}^{\sigma}\left[\tilde{g}(t)-\sum_{i=1}^{m} \lambda_{i}(t)\right]\right)\right|_{t=0}=0, \Longrightarrow C_{0}=0$.

Using the value of $C_{0}=0$, then (2.3) becomes

$$
\phi_{p}^{*}\left(\mathfrak{D}^{\sigma}\left[\mathfrak{z}(t)-\sum_{i=1}^{m} \lambda_{i}(t)\right]\right)=-I^{\zeta}\left[\psi^{*}(t, \mathfrak{g}(t))\right] .
$$

Applying the $q$-Laplacian operator further on (2.4) we get the form

$$
{ }^{c} \mathscr{D}^{\sigma}\left[\mathfrak{z}(t)-\sum_{i=1}^{m} \lambda_{i}(t)\right]=-\phi_{q}^{*}\left(I^{\zeta}\left[\psi^{*}(t, \mathfrak{s}(t))\right]\right) .
$$

Again taking the integral operator $I^{\sigma}$ to both sides of (2.5) and using Lemma 1.1, then (2.5) becomes

$$
\mathfrak{g}(t)-\sum_{i=1}^{m} \lambda_{i}(t)=-I^{\sigma}\left(\phi_{q}^{*}\left(I^{\zeta}\left[\psi^{*}(t, \mathfrak{g}(t))\right]\right)\right)+a_{0}+a_{1} t+a_{2} t^{2}+a_{3} t^{3}+\cdots+a_{n-1} t^{n-1}
$$

where $a_{j} \in \mathbb{R}$ for $j=0,1,2, \ldots, n-1$.

Using the boundary conditions $\xi^{j}(0)=\sum_{i=1}^{m} \lambda_{i}^{j}(0)$ for $j=0,2,3,4, \ldots, n-1$, in (2.6), $\Longrightarrow a_{j}=0$ for $j=0,2,3,4, \ldots, n-1$. and $z^{\prime}(1)=\sum_{i=1}^{m} \lambda_{i}^{\prime}(1)$, implies that $a_{1}=$ $\left.I^{\sigma-1}\left(\phi_{q}^{*}\left(I^{\zeta}\left[\psi^{*}(t, \tilde{g}(t))\right]\right)\right)\right|_{t=1}$

Putting the values of the constants $a_{i}$ in (2.6), we get

$$
\tilde{\jmath}(t)=\sum_{i=1}^{m} \lambda_{i}(t)+\int_{0}^{1} \mathcal{H}^{\sigma}(t, s) \phi_{q}^{*}\left(\frac{1}{\Gamma(\zeta)} \int_{0}^{\lrcorner}(s-\epsilon)^{\zeta-1} \psi^{*}(\epsilon, \tilde{\jmath}(\epsilon)) d \epsilon\right) d s,
$$

where $\mathscr{H}^{\sigma}(t, s)$ is defined in (2.2).

Lemma 2.2 The Green function $\mathcal{H}^{\sigma}(t, s)$ defined in (2.2) satisfies the following conditions:

$\left(\mathscr{B}_{1}\right) \mathscr{H}^{\sigma}(t, s)>0 \forall s, t \in(0,1)$;

$\left(\mathscr{B}_{2}\right)$ the function $\mathcal{H}^{\sigma}(t, s)$ is increasing and $\mathscr{H}^{\sigma}(1, s)=\max _{t \in[0,1]} \mathcal{H}^{\sigma}(t, s)$;

$\left(\mathscr{B}_{3}\right) \mathcal{H}^{\sigma}(1, s) \geq t^{\sigma-1} \max _{t \in[0,1]} \mathscr{H}^{\sigma}(t, s)$ for $t, s \in(0,1)$. 
Proof To prove $\left(\mathscr{B}_{1}\right)$, we take two cases $\forall t, s \in(0,1)$.

Case 1. For $s \leq t$. As $\sigma>3$, then

$$
\begin{aligned}
\mathcal{H}^{\sigma}(t, s) & =\frac{-(t-s)^{\sigma-1}}{\Gamma(\sigma)}+t \frac{(1-s)^{\sigma-2}}{\Gamma(\sigma-1)} \\
& =\frac{-t^{\sigma-1}\left(1-\frac{s}{t}\right)^{\sigma-1}}{\Gamma(\sigma)}+t \frac{(1-s)^{\sigma-2}}{\Gamma(\sigma-1)} \\
& \geq \frac{-t^{\sigma-1}(1-s)^{\sigma-1}}{\Gamma(\sigma)}+t^{\sigma-1} \frac{(1-s)^{\sigma-2}}{\Gamma(\sigma-1)}>0 .
\end{aligned}
$$

Case 2. When $t \leq s$, we evaluate

$$
\mathcal{H}^{\sigma}(t, s)=t \frac{(1-s)^{\sigma-2}}{\Gamma(\sigma-1)}>0
$$

From (2.8) and (2.9), it is proved that $\mathscr{H}^{\sigma}(t, s)>0 \forall s, t \in(0,1)$.

To prove the condition $\left(\mathscr{B}_{2}\right)$, we assume that $\forall s, t \in(0,1)$.

Case 1. For $s \leq t$. As $\sigma>3$, then

$$
\begin{aligned}
\frac{\partial}{\partial t} \mathcal{H}^{\sigma}(t, s) & =\frac{-(t-s)^{\sigma-2}}{\Gamma(\sigma-1)}+\frac{(1-s)^{\sigma-2}}{\Gamma(\sigma-1)} \\
& =\frac{-t^{\sigma-2}\left(1-\frac{s}{t}\right)^{\sigma-2}}{\Gamma(\sigma-1)}+\frac{(1-s)^{\sigma-2}}{\Gamma(\sigma-1)} \\
& \geq \frac{-t^{\sigma-2}(1-s)^{\sigma-2}}{\Gamma(\sigma-1)}+t^{\sigma-2} \frac{(1-s)^{\sigma-2}}{\Gamma(\sigma-1)}>0 .
\end{aligned}
$$

Case 2. When $t \leq s$, we find that

$$
\frac{\partial}{\partial t} \mathcal{H}^{\sigma}(t, s)=\frac{(1-s)^{\sigma-2}}{\Gamma(\sigma-1)}>0
$$

From Eqs. (2.10) and (2.11), it is shown that $\frac{\partial}{\partial t} \mathcal{H}^{\sigma}(t, s)>0 \forall s, t \in(0,1)$, consequently, $\frac{\partial}{\partial t} \mathcal{H}^{\sigma}(t, s)$ is an increasing function. Thus, we have for $t \geq s$

$$
\max _{t \in[0,1]} \mathcal{H}^{\sigma}(t, s)=\frac{-(1-s)^{\sigma-1}}{\Gamma(\sigma)}+\frac{(1-s)^{\sigma-2}}{\Gamma(\sigma-1)}=\mathscr{H}^{\sigma}(1, s),
$$

and for $s \geq t$

$$
\max _{t \in[0,1]} \mathcal{H}^{\sigma}(t, s)=\frac{(1-s)^{\sigma-2}}{\Gamma(\sigma-1)}=\mathscr{H}^{\sigma}(1, s) .
$$

To prove the condition $\left(\mathscr{B}_{3}\right)$, we assume that 
Case 1. For $s \leq t$. As $\sigma>3$, then

$$
\begin{aligned}
\mathcal{H}^{\sigma}(t, s) & =\frac{-(t-s)^{\sigma-1}}{\Gamma(\sigma)}+t \frac{(1-s)^{\sigma-2}}{\Gamma(\sigma-1)} \\
& =\frac{-t^{\sigma-1}\left(1-\frac{s}{t}\right)^{\sigma-1}}{\Gamma(\sigma)}+t \frac{(1-s)^{\sigma-2}}{\Gamma(\sigma-1)} \\
& \geq \frac{-t^{\sigma-1}(1-s)^{\sigma-1}}{\Gamma(\sigma)}+t^{\sigma-1} \frac{(1-s)^{\sigma-2}}{\Gamma(\sigma-1)} \\
& =t^{\sigma-1}\left(\frac{-(1-s)^{\sigma-1}}{\Gamma(\sigma)}+\frac{(1-s)^{\sigma-2}}{\Gamma(\sigma-1)}\right)=t^{\sigma-1} \mathcal{H}^{\sigma}(1, s) .
\end{aligned}
$$

Case 2. For $t \leq s$, we evaluate

$$
\begin{aligned}
\mathcal{H}^{\sigma}(t, s) & =t \frac{(1-s)^{\sigma-2}}{\Gamma(\sigma-1)} \\
& \geq t^{\sigma-1} \frac{(1-s)^{\sigma-2}}{\Gamma(\sigma-1)}=t^{\sigma-1} \mathcal{H}^{\sigma}(1, s) .
\end{aligned}
$$

Thus, by Eqs. (2.14) and (2.15), condition $\mathscr{B}_{3}$ is proved.

\section{Existence result}

Now we prove our existence result by introducing the following conditions.

Let $\Omega^{*}=C[0,1]$ be the Banach space containing all real valued functions defined on $[0,1]$, which are continuous and endowed with the $\|y\|=\max _{t \in[0,1]}\left\{|z(t)|: \xi \in \Omega^{*}\right\}$. Suppose that $\mathcal{K}^{*} \in \Omega^{*}$ is a cone of functions, which are positive and of the type $\mathcal{K}^{*}=\{z \in$ $\left.\Omega^{*}: \xi(t) \geq t^{\sigma}\|z\|, t \in[0,1]\right\}$. Let $\mathcal{A}^{*}(r)=\left\{z \in \mathcal{K}^{*}:\|z\|<r\right\}, \partial \mathcal{A}^{*}(r)=\left\{z \in \mathcal{K}^{*}:\|z\|=r\right\}$. By using Theorem 2.1. Equation (1.1) is equivalent to

$$
\xi(t)=\sum_{i=1}^{m} \lambda_{i}(t)+\int_{0}^{1} \mathcal{H}^{\sigma}(t, s) \phi_{q}^{*}\left(\frac{1}{\Gamma(\zeta)} \int_{0}^{s}(s-\epsilon)^{\zeta-1} \psi^{*}(\epsilon, \xi(\epsilon)) d \epsilon\right) d s .
$$

Let us define an operator $\mathcal{L}^{*}: \mathcal{K}^{*} \backslash\{0\} \rightarrow \Omega^{*}$ associated with problem (1.1), such that

$$
\mathscr{L}^{*} \mathfrak{y}(t)=\sum_{i=1}^{m} \lambda_{i}(t)+\int_{0}^{1} \mathcal{H}^{\sigma}(t, s) \phi_{q}^{*}\left(\frac{1}{\Gamma(\zeta)} \int_{0}^{s}(s-\epsilon)^{\zeta-1} \psi^{*}(\epsilon, \mathfrak{z}(\epsilon)) d \epsilon\right) d s .
$$

By using Theorem 2.1, the solution of FDE given by Eq. (1.1) is a fixed point $y(t)$ of $\mathcal{L}^{*}$ i.e.,

$$
y(t)=\mathcal{L}^{*} y(t) .
$$

To obtain the existence result we need the following assumptions:

$\left(\mathscr{R}_{1}\right) \psi^{*}(t, \xi(t)):[0,1] \times(0,+\infty) \longrightarrow \mathbb{R}^{+}$is a continuous function.

$\left(\mathscr{R}_{2}\right) \lambda_{i}(t):[0,1] \longrightarrow \mathbb{R}^{+}$are also continuous functions for each $i=1,2,3, \ldots, m$, with

$$
\sum_{i=1}^{m} \lambda_{i}(t) \| \leq \bar{\Delta}<+\infty
$$


$\left(R_{3}\right)\left|\psi^{*}(t, \mathfrak{z}(t))\right| \leq \phi_{p}^{*}\left(\Lambda_{1}|\mathfrak{z}(t)|^{l_{1}}+\Lambda_{2}\right) \forall t \in[0,1], \mathfrak{z} \in \Omega^{*}$ where $\Lambda_{1}, \Lambda_{2}$ are positive constants and $l_{1} \in[0,1]$.

$\left(R_{4}\right)\left|\psi^{*}(t, \mathfrak{z})-\psi^{*}(t, v)\right| \leq \mathcal{L}(|\mathfrak{z}-v|) \forall \mathcal{L}>0, t \in[0,1], \mathcal{L}>0, \mathfrak{z}, v \in \Omega^{*}$.

Theorem 3.1 Let us assume that conditions $\left(\mathscr{R}_{1}\right)-\left(\mathscr{R}_{3}\right)$ are satisfied. Then $\mathcal{L}^{*}$ is a completely continuous operator.

Proof For any $\left.z \in\left(\overline{\mathcal{A}^{*}{ }_{2}(r)}\right) \backslash \mathcal{A}_{1}^{*}(r)\right)$, using Lemma 2.2 and (3.2), we have

$$
\begin{aligned}
\mathcal{L}^{*} \mathfrak{z}(t) & =\sum_{i=1}^{m} \lambda_{i}(t)+\int_{0}^{1} \mathcal{H}^{\sigma}(t, s) \phi_{q}^{*}\left(\frac{1}{\Gamma(\zeta)} \int_{0}^{\lrcorner}(s-\epsilon)^{\zeta-1} \psi^{*}(\epsilon, \mathfrak{\jmath}(\epsilon)) d \epsilon\right) d s \\
& \leq \sum_{i=1}^{m} \lambda_{i}(t)+\int_{0}^{1} \mathcal{H}^{\sigma}(1, s) \phi_{q}^{*}\left(\frac{1}{\Gamma(\zeta)} \int_{0}^{\lrcorner}(s-\epsilon)^{\zeta-1} \psi^{*}(\epsilon, \mathfrak{\jmath}(\epsilon)) d \epsilon\right) d s, \\
\mathcal{L}^{*} \mathfrak{g}(t) & =\sum_{i=1}^{m} \lambda_{i}(t)+\int_{0}^{1} \mathcal{H}^{\sigma}(t, s) \phi_{q}^{*}\left(\frac{1}{\Gamma(\zeta)} \int_{0}^{s}(s-\epsilon)^{\zeta-1} \psi^{*}(\epsilon, \mathfrak{\jmath}(\epsilon)) d \epsilon\right) d s \\
& \geq \sum_{i=1}^{m} \lambda_{i}(t)+t^{\sigma-1} \int_{0}^{1} \mathcal{H}^{\sigma}(1, \jmath) \phi_{q}^{*}\left(\frac{1}{\Gamma(\zeta)} \int_{0}^{s}(s-\epsilon)^{\zeta-1} \psi^{*}(\epsilon, \mathfrak{g}(\epsilon)) d \epsilon\right) d s .
\end{aligned}
$$

From (3.4) and (3.5), we arrive at

$$
\mathcal{L}^{*} \mathfrak{z}(t) \geq t^{\sigma-1}\left\|\mathcal{L}^{*} \mathfrak{z}(t)\right\|, \quad t \in[0,1] .
$$

This implies that $\left.\mathcal{L}^{*}:\left(\overline{\mathcal{A}^{*}{ }_{2}(r)}\right) \backslash \mathcal{A}_{1}^{*}(r)\right) \rightarrow \mathcal{K}^{*}$.

Next, to show that $\mathcal{L}^{*}$ is a continuous map, we prove that $\left\|\mathcal{L}^{*} \xi_{n}(t)-\mathcal{L}^{*} \mathfrak{z}(t)\right\| \longrightarrow 0$ as $n \longrightarrow \infty$; let us address

$$
\begin{aligned}
& \left\|\mathcal{L}^{*} z_{n}(t)-\mathcal{L}^{*} \mathfrak{z}(t)\right\| \\
& =\mid \sum_{i=1}^{m} \lambda_{i}(t)+\int_{0}^{1} \mathcal{H}^{\sigma}(t, s) \phi_{q}^{*}\left(\frac{1}{\Gamma(\zeta)} \int_{0}^{s}(s-\epsilon)^{\zeta-1} \psi^{*}\left(\epsilon, \mathfrak{g}_{n}(\epsilon)\right) d \epsilon\right) d s \\
& -\sum_{i=1}^{m} \lambda_{i}(t)-\int_{0}^{1} \mathscr{H}^{\sigma}(t, s) \phi_{q}^{*}\left(\frac{1}{\Gamma(\zeta)} \int_{0}^{s}(s-\epsilon)^{\zeta-1} \psi^{*}(\epsilon, \xi(\epsilon)) d \epsilon\right) d s \mid \\
& \leq \int_{0}^{1}\left|\mathcal{H}^{\sigma}(t, s)\right| \mid \phi_{q}^{*}\left(\frac{1}{\Gamma(\zeta)} \int_{0}^{s}(s-\epsilon)^{\zeta-1} \psi^{*}\left(\epsilon, \check{\zeta}_{n}(\epsilon)\right) d \epsilon\right) d s \\
& \left.-\phi_{q}^{*}\left(\frac{1}{\Gamma(\zeta)} \int_{0}^{\lrcorner}(s-\epsilon)^{\zeta-1} \psi^{*}(\epsilon, \xi(\epsilon)) d \epsilon\right) d\right\lrcorner \mid .
\end{aligned}
$$

By continuity of the function $\psi^{*}$, we have $\left\|\mathcal{L}^{*} \xi_{n}(t)-\mathcal{L}^{*} \xi^{\prime}(t)\right\| \longrightarrow 0$ as $n \longrightarrow \infty$. This implies that $\mathcal{L}^{*}$ is a continuous map.

Now, we have to prove $\mathcal{G}^{*}$ is uniformly bounded on $\left(\overline{\mathcal{A}^{*}{ }_{2}(r)}\right) \backslash \mathcal{A}^{*}{ }_{1}(r)$. 
By (3.2) and using $\left(\mathscr{R}_{2} \mathscr{R}_{3}\right)$, for any $t \in[0,1]$, we get

$$
\begin{aligned}
\left\|\mathcal{L}^{*} \xi\right\| & \\
& =\left|\sum_{i=1}^{m} \lambda_{i}(t)+\int_{0}^{1} \mathcal{H}^{\sigma}(t, s) \phi_{q}^{*}\left(\frac{1}{\Gamma(\zeta)} \int_{0}^{s}(s-\epsilon)^{\zeta-1} \psi^{*}(\epsilon, \mathfrak{\jmath}(\epsilon)) d \epsilon\right) d s\right| \\
& \leq\left|\sum_{i=1}^{m} \lambda_{i}(t)\right|+\int_{0}^{1}\left|\mathcal{H}^{\sigma}(1, s)\right| \phi_{q}^{*}\left(\frac{1}{\Gamma(\zeta)} \int_{0}^{s}(s-\epsilon)^{\zeta-1} \phi_{p}^{*}\left(\Lambda_{1}\|\xi(\epsilon)\|^{l_{1}}+\Lambda_{2}\right) d \epsilon\right) d s \\
& \leq \bar{\Delta}+\left[\frac{1}{\Gamma(\sigma+1)}+\frac{1}{\Gamma(\sigma)}\right]\left[\frac{1}{\Gamma(\zeta+1)}\right]^{q-1}\left(\Lambda_{1}\|\tilde{z}(\epsilon)\|^{l_{1}}+\Lambda_{2}\right) \\
& =\bar{\Delta}+\Theta\left(\Lambda_{1}\|\tilde{\jmath}(\epsilon)\|^{l_{1}}+\Lambda_{2}\right),
\end{aligned}
$$

where $\Theta=\left[\frac{1}{\Gamma(\sigma+1)}+\frac{1}{\Gamma(\sigma)}\right]\left[\frac{1}{\Gamma(\zeta+1)}\right]^{q-1}$.

This proves that $\mathcal{L}^{*}$ is uniformly bounded.

In order to show that the operator $\mathcal{L}^{*}$ is compact, we show the equicontinuity of the operator $\mathcal{G}^{*}$.

For $0<t_{1}<t_{2}<1$, we have

$$
\begin{aligned}
& \left|\mathcal{L}^{*} \mathfrak{z}\left(t_{2}\right)-\mathcal{L}^{*} \mathfrak{z}\left(t_{1}\right)\right| \\
& \leq\left|\sum_{i=1}^{m} \lambda_{i}\left(t_{2}\right)-\sum_{i=1}^{m} \lambda_{i}\left(t_{1}\right)\right| \\
& +\mid \int_{0}^{1} \mathcal{H}^{\sigma}\left(t_{2}, \jmath\right) \phi_{q}^{*}\left(\frac{1}{\Gamma(\zeta)} \int_{0}^{\lrcorner}(\jmath-\epsilon)^{\zeta-1} \psi^{*}(\epsilon, \mathfrak{z}(\epsilon)) d \epsilon\right) d s \\
& -\int_{0}^{1} \mathcal{H}^{\sigma}\left(t_{1}, s\right) \phi_{q}^{*}\left(\frac{1}{\Gamma(\zeta)} \int_{0}^{\lrcorner}(s-\epsilon)^{\zeta-1} \psi^{*}(\epsilon, \tilde{\jmath}(\epsilon)) d \epsilon\right) d s \mid \\
& \leq\left|\sum_{i=1}^{m} \lambda_{i}\left(t_{2}\right)-\sum_{i=1}^{m} \lambda_{i}\left(t_{1}\right)\right| \\
& +\int_{0}^{1}\left|\mathcal{H}^{\sigma}\left(t_{2}, s\right)-\mathcal{H}^{\sigma}\left(t_{1}, s\right)\right| \phi_{q}^{*}\left(\frac{1}{\Gamma(\zeta)} \int_{0}^{s}(s-\epsilon)^{\zeta-1} \phi_{p}^{*}\left(\Lambda_{1}\|\mathfrak{z}(\epsilon)\|^{l_{1}}+\Lambda_{2}\right) d \epsilon\right) d s \\
& \leq\left(\frac{\left|t_{2}{ }^{\sigma}-t_{1}{ }^{\sigma}\right|}{\Gamma(\sigma+1)}+\frac{\left|t_{2}-t_{1}\right|}{\Gamma(\sigma)}\right)\left[\frac{1}{\Gamma(\zeta+1)}\right]^{q-1}\left(\Lambda_{1}\|\mathfrak{z}(\epsilon)\|^{l_{1}}+\Lambda_{2}\right) \\
& \times\left|\mathcal{L}^{*} \mathfrak{z}\left(t_{2}\right)-\mathcal{L}^{*} \mathfrak{z}\left(t_{1}\right)\right| \\
& \longrightarrow 0 \quad \text { as }\left(t_{2}-t_{1}\right) \longrightarrow 0 \text {. }
\end{aligned}
$$

Thus, $\mathcal{L}^{*}$ is an equicontinuous operator on $\left(\overline{\mathcal{A}^{*} 2(r)}\right) \backslash \mathcal{A}_{1}^{*}(r)$ and by the Arzela-Ascoli theorem $\mathcal{L}^{*}$ is compact on $\left(\overline{\mathcal{A}^{*}(r)}\right) \backslash \mathcal{A}_{1}^{*}(r)$. In fact, all the conditions of Theorem 2.1 [58] are satisfied. Thus $\left.\mathcal{L}^{*}:\left(\overline{\mathcal{A}^{*}(r)}\right) \backslash \mathcal{A}_{1}^{*}(r)\right) \rightarrow \mathscr{K}^{*}$ is a completely continuous operator.

Now here, let us determine the hight functions for $\psi^{*}(t, \mathfrak{z}(t))$ for $r>0, \forall t \in[0,1]$

$$
\left\{\begin{array}{l}
\phi_{\min }^{*}(t, r)=\min _{t \in[0,1]}\left\{\psi^{*}(t, \mathfrak{z}(t)): t^{\sigma-1} r \leq \mathfrak{z} \leq r\right\} \geq \overline{\mathrm{m}}>-\infty, \\
\phi_{\max }^{*}(t, r)=\max _{t \in[0,1]}\left\{\psi^{*}(t, \mathfrak{z}(t)): t^{\sigma-1} r \leq \mathfrak{z} \leq r\right\} \leq \overline{\mathrm{M}}<+\infty .
\end{array}\right.
$$


Theorem 3.2 Suppose that assumptions $\left(\mathscr{R}_{1}\right)-\left(\mathscr{R}_{3}\right)$, are satisfied and $\exists a, b \in \mathbb{R}^{+}$such that any of the following condition is satisfied:

$\left(S_{1}\right) a \leq\left\|\sum_{i=1}^{m} \lambda_{i}(t)\right\|+\int_{0}^{1}\left|\mathcal{H}^{\sigma}(1, s)\right| \phi_{q}^{*}\left(\frac{1}{\Gamma(\zeta)} \int_{0}^{s}(s-\epsilon)^{\zeta-1} \phi_{\min }^{*}(\epsilon, a) d \epsilon\right) d s<+\infty$ and $\left\|\sum_{i=1}^{m} \lambda_{i}(t)\right\|+\int_{0}^{1}\left|\mathcal{H}^{\sigma}(1, s)\right| \phi_{q}^{*}\left(\frac{1}{\Gamma(\zeta)} \int_{0}^{s}(s-\epsilon)^{\zeta-1} \phi_{\max }^{*}(\epsilon, \mathcal{b}) d \epsilon\right) d s \leq \mathcal{b}$, or

$\left(S_{2}\right)\left\|\sum_{i=1}^{m} \lambda_{i}(t)\right\|+\int_{0}^{1}\left|\mathcal{H}^{\sigma}(1, s)\right| \phi_{q}^{*}\left(\frac{1}{\Gamma(\zeta)} \int_{0}^{s}(s-\epsilon)^{\zeta-1} \phi_{\max }^{*}(\epsilon, a) d \epsilon\right) d s<a \quad$ and $b \leq\left\|\sum_{i=1}^{m} \lambda_{i}(t)\right\|+\int_{0}^{1}\left|\mathcal{H}^{\sigma}(1, s)\right| \phi_{q}^{*}\left(\frac{1}{\Gamma(\zeta)} \int_{0}^{s}(s-\epsilon)^{\zeta-1} \phi_{\min }^{*}(\epsilon, b) d \epsilon\right) d s<+\infty$.

Then Eq. (1.1) has a positive solution $z \in \mathscr{K}^{*}$ and $a \leq\|z\| \leq b$.

Proof Firstly, we are considering the case $\left(\mathcal{S}_{1}\right)$. If $z \in \partial \mathcal{A}^{*}(a)$ then $\|z\|=a$ and $\forall t \in[0,1]$, $t^{\sigma-1} a \leq z \leq a$. Using (3.10), $\phi_{\min }^{*}(t, a) \leq \psi^{*}(t, \xi(t))$, we write

$$
\begin{aligned}
\left\|\mathcal{L}^{*} \xi\right\| & =\max _{t \in[0,1]}\left|\sum_{i=1}^{m} \lambda_{i}(t)+\int_{0}^{1} \mathcal{H}^{\sigma}(t, s) \phi_{q}^{*}\left(\frac{1}{\Gamma(\zeta)} \int_{0}^{s}(s-\epsilon)^{\zeta-1} \psi^{*}(\epsilon, \mathfrak{z}(\epsilon)) d \epsilon\right) d s\right| \\
& \geq\left\|\sum_{i=1}^{m} \lambda_{i}(t)\right\|+t^{\sigma-1} \int_{0}^{1}\left|\mathcal{H}^{\sigma}(1, s)\right| \phi_{q}^{*}\left(\frac{1}{\Gamma(\zeta)} \int_{0}^{s}(s-\epsilon)^{\zeta-1} \psi^{*}(\epsilon, \mathfrak{z}(\epsilon)) d \epsilon\right) d s \\
& \geq\left\|\sum_{i=1}^{m} \lambda_{i}(t)\right\|+t^{\sigma-1} \int_{0}^{1}\left|\mathcal{H}^{\sigma}(1, s)\right| \phi_{q}^{*}\left(\frac{1}{\Gamma(\zeta)} \int_{0}^{s}(s-\epsilon)^{\zeta-1} \phi_{\min }^{*}(\epsilon, a) d \epsilon\right) d s \geq a \\
& =\|\mathfrak{z}\| .
\end{aligned}
$$

Now, for all $t \in[0,1], t^{\sigma-1} \mathfrak{b} \leq z \leq b$.

If $\mathfrak{z} \in \partial \mathcal{A}^{*}(\mathfrak{b})$ then $\|\mathfrak{z}\|=\mathfrak{b}$ and, using (3.10), we have $\phi_{\max }^{*}(t, b) \geq \psi^{*}(t, \mathfrak{z}(t))$; we find

$$
\begin{aligned}
\left\|\mathcal{L}^{*} \mathfrak{z}\right\| & =\max _{t \in[0,1]}\left|\sum_{i=1}^{m} \lambda_{i}(t)+\int_{0}^{1} \mathcal{H}^{\sigma}(t, s) \phi_{q}^{*}\left(\frac{1}{\Gamma(\zeta)} \int_{0}^{s}(s-\epsilon)^{\zeta-1} \psi^{*}(\epsilon, \mathfrak{g}(\epsilon)) d \epsilon\right) d s\right| \\
& \leq\left\|\sum_{i=1}^{m} \lambda_{i}(t)\right\|+\int_{0}^{1}\left|\mathcal{H}^{\sigma}(1, s)\right| \phi_{q}^{*}\left(\frac{1}{\Gamma(\zeta)} \int_{0}^{s}(s-\epsilon)^{\zeta-1} \psi^{*}(\epsilon, \mathfrak{z}(\epsilon)) d \epsilon\right) d s \\
& \leq\left\|\sum_{i=1}^{m} \lambda_{i}(t)\right\|+t^{\sigma-1} \int_{0}^{1}\left|\mathcal{H}^{\sigma}(1, s)\right| \phi_{q}^{*}\left(\frac{1}{\Gamma(\zeta)} \int_{0}^{s}(s-\epsilon)^{\zeta-1} \phi_{\min }^{*}(\epsilon, a) d \epsilon\right) d s \geq a \\
& =\|\mathfrak{z}\| .
\end{aligned}
$$

Using Lemma 1.2, $z \in\left(\overline{\mathcal{A}^{*}(\mathcal{b})}\right) \backslash \mathcal{A}^{*}(a)$ is a fixed point of $\mathcal{L}^{*}$. By using Lemma 2.2 and Theorem 2.1, for $t \in(0,1)$ and $a \leq\|\mathfrak{z}\| \leq \mathfrak{b}$, we have $\mathfrak{g}(t) \geq t^{\sigma-1}\|\mathfrak{g}(t)\| \geq a t^{\sigma-1}>0$. Therefore $\mathfrak{z}(t)$ is positive solution. It obeys

$$
\begin{aligned}
\frac{\partial}{\partial t} \mathfrak{z}(t) & =\frac{\partial}{\partial t} \mathcal{L}^{*} \mathfrak{z}(t) \\
& =\frac{\partial}{\partial t} \sum_{i=1}^{m} \lambda_{i}(t)+\int_{0}^{1} \frac{\partial}{\partial t} \mathcal{H}^{\sigma}(t, s) \phi_{q}^{*}\left(\frac{1}{\Gamma(\zeta)} \int_{0}^{s}(s-\epsilon)^{\zeta-1} \psi^{*}(\epsilon, \mathfrak{\jmath}(\epsilon)) d \epsilon\right) d s \\
& >0 .
\end{aligned}
$$




\subsection{Uniqueness result}

Theorem 3.3 Let us assume that assumptions $\left(\mathscr{R}_{1}\right),\left(\mathscr{R}_{2}\right)$ and $\left(\mathscr{R}_{4}\right)$ are satisfied. Then there exists a unique solution for Eq. (1.1) on [0,1], if

$$
\Delta^{*}=\mathcal{L}(q-1)\left[\frac{\overline{\mathrm{M}}}{\Gamma(\zeta+1)}\right]^{q-2}\left[\frac{1}{\Gamma(\sigma+1)}+\frac{1}{\Gamma(\sigma)}\right]\left[\frac{1}{\Gamma(\zeta+1)}\right]^{q-1} \leq 1
$$

Proof We prove the uniqueness result for $p \geq 2$.

By (3.10) and, for all $t \in[0,1]$,

$$
\begin{aligned}
I^{\zeta}\left[\psi^{*}(t, z(t))\right] & =\frac{1}{\Gamma(\zeta)} \int_{0}^{t}(t-s)^{\zeta-1} \psi^{*}(s, z(s)) d s \\
& \leq \frac{1}{\Gamma(\zeta)} \int_{0}^{t}(t-s)^{\zeta-1} \overline{\mathrm{M}} d s \leq \frac{\overline{\mathrm{M}}}{\Gamma(\zeta+1)}
\end{aligned}
$$

For each $z \in\left(\overline{\mathcal{A}^{*}(r)}\right) \backslash \mathcal{A}^{*}(r)$ and using (3.15) we have

$$
\begin{aligned}
\| \mathcal{L}^{*} \xi & -\mathcal{L}^{*} v \| \\
= & \max _{t \in[0,1]} \mid \sum_{i=1}^{m} \lambda_{i}(t)+\int_{0}^{1} \mathcal{H}^{\sigma}(t, s) \phi_{q}^{*}\left(\frac{1}{\Gamma(\zeta)} \int_{0}^{s}(s-\epsilon)^{\zeta-1} \psi^{*}(\epsilon, \mathfrak{s}(\epsilon)) d \epsilon\right) d s \\
& -\sum_{i=1}^{m} \lambda_{i}(t)-\int_{0}^{1} \mathcal{H}^{\sigma}(t, s) \phi_{q}^{*}\left(\frac{1}{\Gamma(\zeta)} \int_{0}^{s}(s-\epsilon)^{\zeta-1} \psi^{*}(\epsilon, v(\epsilon)) d \epsilon\right) d s \mid \\
\leq & \int_{0}^{1}\left|\mathcal{H}^{\sigma}(1, s)\right| \phi_{q}^{*}\left(\frac{1}{\Gamma(\zeta)} \int_{0}^{s}(s-\epsilon)^{\zeta-1} \psi^{*}(\epsilon, \xi(\epsilon)) d \epsilon\right) d s \\
& -\phi_{q}^{*}\left(\frac{1}{\Gamma(\zeta)} \int_{0}^{s}(s-\epsilon)^{\zeta-1} \psi^{*}(\epsilon, v(\epsilon)) d \epsilon\right) d s \mid \\
\leq & (q-1)\left[\frac{\overline{\mathrm{M}}}{\Gamma(\zeta+1)}\right]^{q-2} \int_{0}^{1}\left|\mathcal{H}^{\sigma}(1, s)\right| \mid\left(\frac{1}{\Gamma(\zeta)} \int_{0}^{s}(s-\epsilon)^{\zeta-1} \psi^{*}(\epsilon, \mathfrak{z}(\epsilon)) d \epsilon\right) d s \\
& -\left(\frac{1}{\Gamma(\zeta)} \int_{0}^{s}(s-\epsilon)^{\zeta-1} \psi^{*}(\epsilon, v(\epsilon)) d \epsilon\right) d s \mid \\
\leq & \mathcal{L}(q-1)\left[\frac{\bar{M}}{\Gamma(\zeta+1)}\right]^{q-2}\left[\frac{1}{\Gamma(\sigma+1)}+\frac{1}{\Gamma(\sigma)}\right]\left[\frac{1}{\Gamma(\zeta+1)}\right]^{q-1}\|\xi-v\| \\
= & \Delta^{*} \forall t \in[0,1],
\end{aligned}
$$

but in (3.14) we assumed that $\Delta^{*}<1$. This proves that $\mathcal{G}^{*}$ is a contraction map. Hence by the Banach contraction mapping principle there exists a unique fixed point for operator $\mathcal{G}^{*}$. Hence, there exists a unique solution of Eq. $(1.1)$ on $[0,1]$.

\section{Hyers-Ulam stability}

Here, we analyze the HU stability of (1.1). We define the HU stability as follows.

Definition 4.1 ([60]) The integral equation (3.1) is said to be HU stable if there exists a non-negative constant $\Delta^{*}$, for some fixed non-negative constant $\gamma^{*}>0$ satisfying the following: 
If

$$
\left|z(t)-\sum_{i=1}^{m} \lambda_{i}(t)+\int_{0}^{1} \mathcal{H}^{\sigma}(t, s) \phi_{q}^{*}\left(\frac{1}{\Gamma(\zeta)} \int_{0}^{s}(s-\epsilon)^{\zeta-1} \psi^{*}(\epsilon, \xi(\epsilon)) d \epsilon\right) d s\right| \leq \gamma^{*},
$$

then there exists a function $v(t)$, which is continuous and satisfies

$$
v(t)=\sum_{i=1}^{m} \lambda_{i}(t)+\int_{0}^{1} \mathcal{H}^{\sigma}(t, s) \phi_{q}^{*}\left(\frac{1}{\Gamma(\zeta)} \int_{0}^{s}(s-\epsilon)^{\zeta-1} \psi^{*}(\epsilon, v(\epsilon)) d \epsilon\right) d s,
$$

with

$$
|\mathfrak{z}(t)-v(t)| \leq \Delta^{*} \gamma^{*}
$$

Theorem 4.1 The FDE (1.1) with $\phi_{p}^{*}$ operator is HU stable for $p>2$ provided that $\left(\Re_{1}\right)$, $\left(\mathscr{R}_{2}\right)$ and $\left(\mathscr{R}_{4}\right)$ are satisfied.

Proof For the HU stability of the problem (1.1), we prove that for the integral equation (3.1), with assumptions $\left(\mathscr{R}_{1}\right),\left(\mathscr{R}_{2}\right)$ and $\left(\mathscr{R}_{4}\right)$. we have

$$
\begin{aligned}
\mid \xi(t) & -v(t) \mid \\
= & \max _{t \in[0,1]} \mid \sum_{i=1}^{m} \lambda_{i}(t)+\int_{0}^{1} \mathcal{H}^{\sigma}(t, s) \phi_{q}^{*}\left(\frac{1}{\Gamma(\zeta)} \int_{0}^{s}(s-\epsilon)^{\zeta-1} \psi^{*}(\epsilon, \xi(\epsilon)) d \epsilon\right) d s \\
& -\sum_{i=1}^{m} \lambda_{i}(t)-\int_{0}^{1} \mathcal{H}^{\sigma}(t, s) \phi_{q}^{*}\left(\frac{1}{\Gamma(\zeta)} \int_{0}^{s}(s-\epsilon)^{\zeta-1} \psi^{*}(\epsilon, v(\epsilon)) d \epsilon\right) d s \mid \\
\leq & \int_{0}^{1}\left|\mathcal{H}^{\sigma}(1, s)\right| \phi_{q}^{*}\left(\frac{1}{\Gamma(\zeta)} \int_{0}^{s}(s-\epsilon)^{\zeta-1} \psi^{*}(\epsilon, \xi(\epsilon)) d \epsilon\right) d s \\
& -\phi_{q}^{*}\left(\frac{1}{\Gamma(\zeta)} \int_{0}^{s}(s-\epsilon)^{\zeta-1} \psi^{*}(\epsilon, v(\epsilon)) d \epsilon\right) d s \mid \\
\leq & (q-1)\left[\frac{\overline{\mathrm{M}}}{\Gamma(\zeta+1)}\right]^{q-2} \int_{0}^{1}\left|\mathcal{H}^{\sigma}(1, s)\right|\left(\frac{1}{\Gamma(\zeta)} \int_{0}^{s}(s-\epsilon)^{\zeta-1} \psi^{*}(\epsilon, \xi(\epsilon)) d \epsilon\right) d s \\
& -\left(\frac{1}{\Gamma(\zeta)} \int_{0}^{s}(s-\epsilon)^{\zeta-1} \psi^{*}(\epsilon, v(\epsilon)) d \epsilon\right) d s \mid \\
\leq & \mathcal{L}(q-1)\left[\frac{\overline{\mathrm{M}}}{\Gamma(\zeta+1)}\right]^{q-2}\left[\frac{1}{\Gamma(\sigma+1)}+\frac{1}{\Gamma(\sigma)}\right]\left[\frac{1}{\Gamma(\zeta+1)}\right]^{q-1}\|\xi-v\| \\
= & \Delta^{*} \forall t \in[0,1] .
\end{aligned}
$$

Hence using (4.4) Eq. (3.1) is HU stable. As a result, the FDE (1.1) is HU stable.

\section{Example}

Here, we present some examples to illustrate our results. 
Example 5.1 Let us take the following FDE:

$$
\left\{\begin{array}{l}
{ }^{c} \mathscr{D}^{\zeta} \phi_{p}^{*}\left[{ }^{c} \mathscr{D}^{\sigma}\left(\mathfrak{z}(t)-\sum_{i=1}^{m} \lambda_{i}(t)\right)\right]=-\psi^{*}(t, \mathfrak{z}(t)), \quad t \in[0,1], \\
\left.\phi_{p}^{*}\left[{ }^{c} \mathscr{D}^{\sigma} \mathfrak{g}(t)-\sum_{i=1}^{m} \lambda_{i}(t)\right]\right|_{t=0}=0, \quad \mathfrak{g}(0)=\sum_{i=1}^{m} \lambda_{i}(0), \\
\mathfrak{g}^{\prime}(1)=\sum_{i=1}^{m} \lambda_{i}^{\prime}(1), \quad \mathfrak{g}^{j}(0)=\sum_{i=1}^{m} \lambda_{i}^{j}(0) \quad \text { for } j=2,3,4, \ldots, n-1,
\end{array}\right.
$$

where $\zeta=0.5, \sigma=3.6, p=5, q=1.25, m=3$. We have $\sum_{i=1}^{m} \lambda_{i}(t)=\frac{1}{t^{2}+100+i} \forall t \in[0,1]$, $\psi^{*}(t, \mathfrak{z}(t))=\frac{1}{t^{2}+20}\left[|y|^{5}+\frac{1}{\left(1+7|\mathfrak{y}|^{\frac{5}{39}}\right)}\right]$.

Let us consider

$$
\begin{aligned}
\bar{M} & =\phi_{\max }^{*}(t, r)=\max _{t \in[0,1]}\left\{\frac{1}{t^{2}+20}\left[|z|^{5}+\frac{1}{\left(1+7|\mathfrak{z}|^{\frac{5}{39}}\right)}\right]: t^{\frac{13}{5}} r \leq z \leq r\right\} \\
& \leq \max _{t \in[0,1]}\left(\frac{1}{t^{2}+20}\left[|r|^{5}+\frac{1}{\left(1+7\left|t^{\frac{1}{3}} \frac{5}{3^{39}}\right|\right.}\right]\right) \\
& \leq 0.1 \quad \forall t \in[0,1], r=b=1, \\
\phi_{\min }^{*}(t, r) & =\min _{t \in[0,1]}\left\{\frac{1}{t^{2}+20}\left[|\mathfrak{z}|^{5}+\frac{1}{\left(1+7|\mathfrak{z}|^{\frac{5}{39}}\right)}\right]: t^{\frac{13}{5}} r \leq z \leq r\right\} \\
& \geq \min _{t \in[0,1]}\left(\frac{1}{t^{2}+20}\left[\left|t^{13} r^{5}\right|+\frac{1}{\left(1+7\left|r^{\frac{5}{39}}\right|\right.}\right]\right) \\
& \geq 0.0122503 \quad \forall t \in[0,1], r=a=\frac{1}{1000} .
\end{aligned}
$$

Then

$$
\begin{aligned}
& \max _{t \in[0,1]}\left(\sum_{i=1}^{m} \lambda_{i}(t)+\int_{0}^{1} \mathcal{H}^{\sigma}(t, s) \phi_{q}^{*}\left(\frac{1}{\Gamma(\zeta)} \int_{0}^{s}(s-\epsilon)^{\zeta-1} \psi^{*}(\epsilon, \mathfrak{\jmath}(\epsilon)) d \epsilon\right) d s\right) \\
& \leq 0.115663+\int_{0}^{1} \mathcal{H}^{\sigma}(1, s) \phi_{q}^{*}\left(\frac{1}{\Gamma(\zeta)} \int_{0}^{s}(s-\epsilon)^{\zeta-1} \phi_{\max }^{*}(t, b) d \epsilon\right) d s \\
& \leq 0.115663+\int_{0}^{1} \mathcal{H}^{\sigma}(1, s) \phi_{q}^{*}\left(\frac{1}{\Gamma(\zeta)} \int_{0}^{s}(s-\epsilon)^{\zeta-1} \phi_{\max }^{*}(t, 1) d \epsilon\right) d s \\
& \leq 0.115663+\int_{0}^{1} \mathcal{H}^{\sigma}(1, s) \phi_{q}^{*}\left(\frac{1}{\Gamma(\zeta)} \int_{0}^{s}(s-\epsilon)^{\zeta-1} \phi_{\max }^{*}(t, 1) d \epsilon\right) d s \\
& \quad \leq 0.115663+\overline{\mathrm{M}}^{q-1}\left[\frac{1}{\Gamma(\sigma+1)}+\frac{1}{\Gamma(\sigma)}\right]\left[\frac{1}{\Gamma(\zeta+1)}\right]^{q-1} \\
& \quad \leq 0.314901<1 .
\end{aligned}
$$

Also we have

$$
\begin{gathered}
\min _{t \in[0,1]}\left(\sum_{i=1}^{m} \lambda_{i}(t)+\int_{0}^{1} \mathcal{H}^{\sigma}(t, s) \phi_{q}^{*}\left(\frac{1}{\Gamma(\zeta)} \int_{0}^{s}(s-\epsilon)^{\zeta-1} \psi^{*}(\epsilon, \mathfrak{z}(\epsilon)) d \epsilon\right) d s\right) \\
\geq 0.029414+\int_{0}^{1} \mathcal{H}^{\sigma}(1, s) \phi_{q}^{*}\left(\frac{1}{\Gamma(\zeta)} \int_{0}^{s}(s-\epsilon)^{\zeta-1} \phi_{\min }^{*}(t, a) d \epsilon\right) d s
\end{gathered}
$$




$$
\begin{aligned}
& \geq 0.029414+\int_{0}^{1} \mathcal{H}^{\sigma}(1, s) \phi_{q}^{*}\left(\frac{1}{\Gamma(\zeta)} \int_{0}^{s}(s-\epsilon)^{\zeta-1} \phi_{\min }^{*}\left(t, \frac{1}{1000}\right) d \epsilon\right) d s \\
& \geq 0.029414>\frac{1}{1000} .
\end{aligned}
$$

Using Theorem 3.2, Eq. (5.1) has a solution $\mathfrak{z}^{*}$ which satisfies $\frac{1}{1000} \leq\|\mathfrak{z}\| \leq 1$.

Example 5.2 Let us take the following FDE:

$$
\left\{\begin{array}{l}
\mathscr{D}^{\zeta} \phi_{p}^{*}\left[\mathscr{D}^{\sigma}\left(\mathfrak{z}(t)-\sum_{i=1}^{m} \lambda_{i}(t)\right)\right]=-\psi^{*}(t, \mathfrak{z}(t)), \quad t \in[0,1], \\
\left.\phi_{p}^{*}\left[{ }^{c} \mathscr{D}^{\sigma} \mathfrak{z}(t)-\sum_{i=1}^{m} \lambda_{i}(t)\right]\right|_{t=0}=0, \quad \mathfrak{z}(0)=\sum_{i=1}^{m} \lambda_{i}(0), \\
\mathfrak{g}^{\prime}(1)=\sum_{i=1}^{m} \lambda_{i}^{\prime}(1), \quad \mathfrak{g}^{j}(0)=\sum_{i=1}^{m} \lambda_{i}^{j}(0) \quad \text { for } j=2,3,4, \ldots, n-1,
\end{array}\right.
$$

where $\zeta=0.5, \sigma=3.5, p=5, q=1.25, m=3$. We have $\sum_{i=1}^{m} \lambda_{i}(t)=\frac{1}{t+100+i} \forall t \in[0,1]$. $\psi^{*}(t, \mathfrak{z}(t))=\frac{1}{t^{2}+20}\left[\frac{|\mathfrak{z}|}{(1+|\mathfrak{z}|)}\right]$, which satisfies the assumption $\left(\mathscr{R}_{4}\right)$ and where $\mathcal{L}=\frac{1}{20}$, that is,

$$
\mid \psi^{*}(t, \mathfrak{z}(t))-\psi^{*}\left(t, v(t)\left|\leq \frac{1}{20}\right| \mathfrak{z}-v \mid .\right.
$$

Consider

$$
\begin{aligned}
\bar{M} & =\phi_{\max }^{*}(t, r)=\max _{t \in[0,1]}\left\{\frac{1}{t^{2}+20}\left[\frac{|z|}{(1+|z|)}\right]: t^{\frac{5}{2}} r \leq z \leq r\right\} \\
& \leq \max _{t \in[0,1]}\left(\frac{1}{t^{2}+20}\left[\frac{|r|}{\left(1+\left|t^{\frac{5}{2}} r\right|\right)}\right]\right) \\
& \leq 0.05 \quad \forall t \in[0,1], r=b=1 .
\end{aligned}
$$

Then

$$
\Delta^{*}=\mathcal{L}(q-1)\left[\frac{\overline{\mathrm{M}}}{\Gamma(\zeta+1)}\right]^{q-2}\left[\frac{1}{\Gamma(\sigma+1)}+\frac{1}{\Gamma(\sigma)}\right]\left[\frac{1}{\Gamma(\zeta+1)}\right]^{q-1} \approx 0.04306 \leq 1
$$

Hence there exists a unique solution of Eq. (5.4) on $[0,1]$ by Theorem 3.2. We can easily check all the conditions of Theorem 4.1 are also satisfied. As a result, Eq. (5.4) is HU stable.

\section{Conclusion}

In this investigation, the existence results for general FDEs (1.1) involving a $\phi_{p}^{*}$-Laplacian operator is established by using Guo-Krasnoselskii's fixed point theorem [58]. The uniqueness results are proved by using the Banach contraction mapping principle and HU stability is also evaluated. The properties of the Green function also proved. The validity of our result is illustrated by examples. Also, one can study the multiple solutions, periodic solutions and controllability for the proposed general non-linear FDEs.

\section{Acknowledgements}

The first author acknowledges with gratitude the Council of Scientific and Industrial Research (CSIR)-New Delhi, India, for supporting this research work under grant no. 09/1051(0031)/2019-EMR-1 and the Department of Mathematics and Statistics, Central University of Punjab, Bathinda, India. 
Availability of data and materials

Not applicable.

\section{Competing interests}

The authors declare that there is no conflict of interests regarding the publication of this paper

\section{Authors' contributions}

All the authors have made equally contributions to the publication of this article. All authors read and approved the final manuscript.

\section{Author details}

'Department of Mathematics and Statistics, School of Basic and Applied Sciences, Central University of Punjab, Bathinda, India. ${ }^{2}$ Department of Mathematics, Faculty of Art and Sciences, Cankaya University, Cankaya, Turkey. ${ }^{3}$ Institute of Space Science, Magurele-Bucharest, Magurele-Bucharest, Romania. ${ }^{4}$ Department of Medical Research, China Medical University Hospital, China Medical University, Taichung, Taiwan. ${ }^{5}$ Department of Mathematics and General Sciences, Prince Sultan University, Riyadh, Saudi Arabia.

\section{Publisher's Note}

Springer Nature remains neutral with regard to jurisdictional claims in published maps and institutional affiliations.

\section{Received: 3 March 2020 Accepted: 27 May 2020 Published online: 19 June 2020}

\section{References}

1. Podlubny, I.: Fractional Differential Equations, vol. 198. Academic Press, San Diego (1998). https://doi.org/10.2307/2653160

2. Kilbas, A.A., Srivastava, H.M., Trujillo, J.J.: Theory and Applications of Fractional Differential Equations, vol. 204. Elsevier, Amsterdam (2006)

3. Sabatier, J., Agrawal, O.P., Machado, J.A.T.: Advances in Fractional Calculus, vol. 4. Springer, Dordrecht (2007). https://doi.org/10.1007/978-1-4020-6042-7

4. Mainardi, F.: Fractional Calculus and Waves in Linear Viscoelasticity: An Introduction to Mathematical Models. World Scientific, Singapore (2010). https://doi.org/10.1142/p614

5. Yang, X.J.: General Fractional Derivatives: Theory, Methods and Applications. CRC Press, New York (2019)

6. Gómez-Aguilar, J.F., Atangana, A.: Fractional Hunter-Saxton equation involving partial operators with bi-order in Riemann-Liouville and Liouville-Caputo sense. Eur. Phys. J. Plus 132(2), 1-18 (2017). https://doi.org/10.1140/epjp/i2017-11371-6

7. Yang, X.J., Gao, F., Ju, Y., Zhou, H.E.: Fundamental solutions of the general fractional-order diffusion equations. Math. Methods Appl. Sci. 41, 9312-9320 (2018). https://doi.org/10.1002/mma.5341

8. Atangana, A., Gómez-Aguilar, J.F.: Decolonisation of fractional calculus rules: breaking commutativity and associativity to capture more natural phenomena. Eur. Phys. J. Plus 133, 1-23 (2018). https://doi.org/10.1140/epjp/i2018-12021-3

9. Atangana, A., Hammouch, Z.: Fractional calculus with power law: the cradle of our ancestors. Eur. Phys. J. Plus 134(9), 1-15 (2019). https://doi.org/10.1140/epjp/i2019-12777-8

10. Agarwal, R.P., O'Regan, D., Stanek, S.: Positive solutions for Dirichlet problems of singular nonlinear fractional differential equations. J. Math. Anal. Appl. 371(1), 57-68 (2010). https://doi.org/10.1016/j.jmaa.2010.04.034

11. Agarwal, R.P., O'Regan, D., Stanek, S.: Positive solutions for mixed problems of singular fractional differential equations. Math. Nachr. 285(1), 27-41 (2012). https://doi.org/10.1002/mana.201000043

12. Baleanu, D., Agarwal, R.P., Mohammadi, H., Rezapour, S.: Some existence results for a nonlinear fractional differential equation on partially ordered Banach spaces. Bound. Value Probl. 2013, 112 (2013). http://www.boundaryvalueproblems.com/content/2013/1/112

13. Vong, S.: Positive solutions of singular fractional differential equations with integral boundary conditions. Math. Comput. Model. 57(5-6), 1053-1059 (2013). https://doi.org/10.1016/j.mcm.2012.06.024

14. Abdeljawad, T., Alzabut, J.: On Riemann-Liouville fractional q-difference equations and their application to retarded logistic type model. Math. Methods Appl. Sci. 41(18), 8953-8962 (2018). https://doi.org/10.1002/mma.4743

15. Liu, J.G., Yang, X.J., Feng, Y.Y.: On integrability of the time fractional nonlinear heat conduction equation. J. Geom. Phys. 144, 190-198 (2019). https://doi.org/10.1016/j.geomphys.2019.06.004

16. Liu, J.G., Yang, X.J., Feng, Y.Y.: Analytical solutions of some integral fractional differential-difference equations. Mod. Phys. Lett. B 34(1), 02050009 (2020). https://doi.org/10.1142/S0217984920500098

17. Abdeljawad, T., Baleanu, D., Jarad, F.: Existence and uniqueness theorem for a class of delay differential equations with left and right Caputo fractional derivatives. J. Math. Phys. 49, 083507 (2008). https://doi.org/10.1063/1.2970709

18. Yang, X.J., Feng, Y.Y., Cattani, C., Inc, M.: Fundamental solutions of anomalous diffusion equations with the decay exponential kernel. Math. Methods Appl. Sci. 42, 4054-4060 (2019). https://doi.org/10.1002/mma.5634

19. Saad, K.M., Gómez-Aguilar, J.F.: Analysis of reaction-diffusion system via a new fractional derivative with non-singular kernel. Phys. A, Stat. Mech. Appl. 509, 703-716 (2018). https://doi.org/10.1016/j.physa.2018.05.137

20. Yang, X.J., Gao, F.: A new technology for solving diffusion and heat equations. Therm. Sci. 21(1A), 133-140 (2017)

21. Maraaba, T.A., Jarad, F., Baleanu, D.: On the existence and the uniqueness theorem for fractional differential equations with bounded delay within Caputo derivatives. Sci. China Ser. A, Math. 51, 1775-1786 (2008). https://doi.org/10.1007/s11425-008-0068-1

22. Atangana, A., Gómez-Aguilar, J.F.: Numerical approximation of Riemann-Liouville definition of fractional derivative: from Riemann-Liouville to Atangana-Baleanu. Numer. Methods Partial Differ. Equ. 34(5), 1-22 (2017). https://doi.org/10.1002/num.22195

23. Morales-Delgado, V.F., Gómez-Aguilar, J.F., Taneco-Hernandez, M.A.: Analytical solution of the time fractional diffusion equation and fractional convection-diffusion equation. Rev. Mex. Fis. 65(1), 82-88 (2018) 
24. Atangana, A., Gómez-Aguilar, J.F.: Fractional derivatives with no-index law property: application to chaos and statistics. Chaos Solitons Fractals 114, 516-535 (2018). https://doi.org/10.1016/j.chaos.2018.07.033

25. Gómez-Aguilar, J.F., Yépez-Martínez, H., Escobar-Jiménez, R.F., Olivares-Peregrino, V.H., Reyes, J.M., Sosa, I.O.: Series solution for the time-fractional coupled mkdv equation using the homotopy analysis method. Math. Probl. Eng. 2016, Article ID 7047126 (2016). https://doi.org/10.1155/2016/7047126

26. Saad, K.M., Khader, M.M., Gómez-Aguilar, J.F., Baleanu, D.: Numerical solutions of the fractional Fisher's type equations with Atangana-Baleanu fractional derivative by using spectral collocation methods. Chaos, Interdiscip. J. Nonlinear Sci. 29(2), 1-13 (2019). https://doi.org/10.1063/1.5086771

27. Yang, X.J.: New general fractional-order rheological models with kernels of Mittag-Leffler functions. Rom. Rep. Phys. 69(4), 1-15 (2017)

28. Yang, X.J., Machado, J.A., Nieto, J.J.: A new family of the local fractional PDEs. Fundam. Inform. 151, 63-75 (2017) https://doi.org/10.3233/FI-2017-1479

29. Bhatter, S., Mathur, A., Kumar, D., Singh, J.: A new analysis of fractional Drinfeld-Sokolov-Wilson model with exponential memory. Phys. A, Stat. Mech. Appl. 537, 122578 (2020). https://doi.org/10.1016/j.physa.2019.122578

30. Ravichandran, C., Logeswari, K., Jarad, F.: New results on existence in the framework of Atangana-Baleanu derivative for fractional integro-differential equations. Chaos Solitons Fractals 125, 194-200 (2019). https://doi.org/10.1016/j.chaos.2019.05.014

31. Panda, S.K., Abdeljawad, T., Ravichandran, C.: Novel fixed point approach to Atangana-Baleanu fractional and $L^{P}$-Fredholm integral equations. Alex. Eng. J. 1-12 (2020). https://doi.org/10.1016/j.aej.2019.12.027

32. Yokus, A., Gülbahar, S.: Numerical solutions with linearization techniques of the fractional Harry Dym equation. Appl. Math. Nonlinear Sci. 4(1), 35-42 (2019). https://doi.org/10.2478/AMNS.2019.1.00004

33. Kumar, D., Singh, J., Baleanu, D.: A new numerical algorithm for fractional Fitzhugh-Nagumo equation arising in transmission of nerve impulses. Nonlinear Dyn. 91, 307-317 (2018). https://doi.org/10.1007/s11071-017-3870-x

34. Cattani, C., Ciancio, A.: Existence theorem for hybrid competition model. In: BSG Proceedings 18. The Int. Conf. of Diff. Geom. and Dynamical Systems, vol. 18, pp. 32-38 (2011)

35. Uçar, S., Uçar, E., Özdemir, N., Hammouch, Z:: Mathematical analysis and numerical simulation for a smoking model with Atangana-Baleanu derivative. Chaos Solitons Fractals 118, 300-306 (2019). https://doi.org/10.1016/j.chaos.2018.12.003

36. Jafari, H., Baleanu, D., Khan, H., Khan, R.A., Khan, A.: Existence criterion for the solutions of fractional order p-Laplacian boundary value problems. Bound. Value Probl. 2015(1), 164 (2015). https://doi.org/10.1186/s13661-015-0425-2

37. Bai, C.: Existence and uniqueness of solutions for fractional boundary value problems with p-Laplacian operator. Adv Differ. Equ. 2018(4), 1 (2018). https://doi.org/10.1186/s13662-017-1460-3

38. Yan, R., Sun, S., Lu, H., Zhao, Y.: Existence of solutions for fractional differential equations with integral boundary conditions. Adv. Differ. Equ. 2014(1), 1 (2014). https://doi.org/10.1186/1687-1847-2014-25

39. Li, Y.: Existence of positive solutions for fractional differential equation involving integral boundary conditions with p-Laplacian operator. Adv. Differ. Equ. 2017, 135 (2017) 1-11. https://doi.org/10.1186/s13662-017-1172-8

40. Chen, T., Liu, W., Hu, Z:: A boundary value problem for fractional differential equation with p-Laplacian operator at resonance. Nonlinear Anal. 75, 3210-3217 (2012). https://doi.org/10.1016/j.na.2011.12.020

41. Tan, J.J., Li, M.: Solutions of fractional differential equations with $\mathrm{p}$-Laplacian operator in Banach spaces. Bound. Value Probl. 2018(15), 1 (2018). https://doi.org/10.1186/s13661-018-0930-1

42. Lu, H., Han, Z., Sun, S., Liu, J.: Existence on positive solutions for boundary value problems of nonlinear fractional differential equations with p-Laplacian. Adv. Differ. Equ. 2013, 30 (2013) 1-16. https://doi.org/10.1186/1687-1847-2013-30

43. Khan, H., Jarad, F., Abdeljawad, T., Khan, A.: A singular ABC-fractional differential equation with p-Laplacian operator. Chaos Solitons Fractals 129, 56-61 (2019). https://doi.org/10.1016/j.chaos.2019.08.017

44. Khan, H., Chen, W., Sun, H.: Analysis of positive solution and Hyers-Ulam stability for a class of singular fractional differential equations with p-Laplacian in Banach space. Math. Methods Appl. Sci. 41, 3430-3440 (2018). https://doi.org/10.1002/mma.4835

45. Khan, H., Li, Y., Suna, H., Khan, A.: Existence of solution and Hyers-Ulam stability for a coupled system of fractional differential equations with p-Laplacian operator. Bound. Value Probl. 2017, 157 (2017) 1-16. https://doi.org/10.1186/s13661-017-0878-6

46. Wang, J., Li, X.: Ulam-Hyers stability of fractional Langevin equations. Appl. Math. Comput. 258, 72-83 (2015) https://doi.org/10.1016/j.amc.2015.01.111

47. Khan, A., Gómez-Aguilar, J.F., Khan, T.S., Khan, H.: Stability analysis and numerical solutions of fractional order HIV/AIDS model. Chaos Solitons Fractals 122, 119-128 (2019). https://doi.org/10.1016/j.chaos.2019.03.022

48. Khan, H., Tunc, C., Khan, A.: Stability results and existence theorems for nonlinear delay-fractional differential equations with $\phi_{p}^{*}$-operator. J. Appl. Anal. Comput. 10, 584-597 (2020). https://doi.org/10.11948/20180322

49. Khan, H., Tunc, C., Khan, A.: Green function's properties and existence theorem for nonlinear delay-fractional differential equations. Discrete Contin. Dyn. Syst., Ser. S 13, 1-13 (2020). https://doi.org/10.3934/dcdss.2020139

50. Rizwan, R., Zada, A., Wang, X.: Stability analysis of nonlinear implicit fractional Langevin equation with non instantaneous impulses. Adv. Differ. Equ. 2019, 85 (2019) 1-31. https://doi.org/10.1186/s13662-019-1955-1

51. Khan, H., Gómez-Aguilar, J.F., Khan, A., Khan, T.S.: Stability analysis for fractional order advection-reaction diffusion system. Phys. A, Stat. Mech. Appl. 521, 737-751 (2019). https://doi.org/10.1016/j.physa.2019.01.102

52. Zada, A., Ali, W., Farina, S.: Hyers-Ulam stability of non linear differential equations with fractional integrable impulses. Math. Methods Appl. Sci. 40(15), 5502-5514 (2017). https://doi.org/10.1002/mma.4405

53. Khan, A., Syam, M.I., Zada, A., Khan, H.: Stability analysis of nonlinear fractional differential equations with Caputo and Riemann-Liouville derivatives. Eur. Phys. J. Plus 264,1-9 (2018). https://doi.org/10.1140/epjp/i2018-12119-6

54. Khan, H., Gómez-Aguilar, J.F., Khan, A., Khan, T.S.: Stability analysis for fractional order advection-reaction diffusion system. Phys. A, Stat. Mech. Appl. 521,737-751 (2019). https://doi.org/10.1016/j.physa.2019.01.102

55. Khan, H., Abdeljawad, T., Aslam, M., Khan, R.A., Khan, A.: Existence of positive solution and Hyers Ulam stability for a nonlinear singular-delay-fractional differential equation. Adv. Differ. Equ. 2019, 104 (2019) 1-13.

https://doi.org/10.1186/s13662-019-2054-z 
56. Khan, A., Khan, H., Gómez-Aguilar, J.F., Abdeljawad, T.: Existence and Hyers Ulam stability for a nonlinear singular fractional differential equation with Mittag-Leffler kernel. Chaos Solitons Fractals 127, 422-427 (2019). https://doi.org/10.1016/j.chaos.2019.07.026

57. Khan, H., Khan, A., Abdeljawad, T., Alkhazzan, A.: Existence results in Banach space for a non linear impulsive system. Adv. Differ. Equ. 2019(18), 1 (2019). https://doi.org/10.1186/s13662-019-1965-z

58. Krasnoselsky, M.A.: Two remarks on the method of successive approximation. Usp. Mat. Nauk 10, $123-127$ (1955) http://mi.mathnet.ru/eng/umn7954

59. Guo, D., Lakshmikantham, V.: Nonlinear Problems in Abstract Cones, vol. 5. Academic Press, San Diego (2014)

60. Hyers, D.H.: On the stability of the linear functional equations. Proc. Natl. Acad. Sci. USA 27(4), 222-224 (1941). https://doi.org/10.1073/pnas.27.4.222

Submit your manuscript to a SpringerOpen ${ }^{\circ}$ journal and benefit from:

- Convenient online submission

$\checkmark$ Rigorous peer review

- Open access: articles freely available online

- High visibility within the field

- Retaining the copyright to your article

Submit your next manuscript at $\gg$ springeropen.com 